\title{
Structural Insights into Outer Membrane Protein Biogenesis in Pathogenic Neisseria \\ Evan Billings ${ }^{1}$, Nicholas Noinaj ${ }^{2}$ \\ ${ }^{1}$ Purdue University ${ }^{2}$ Purdue University \\ ebillin@purdue.edu
}

Neisseria gonorrhoeae (Ngo), is an obligate human pathogen and the causative agent of the disease gonorrhea. If left untreated, gonorrhea can lead to serious health issues including ectopic pregnancy and infertility. In addition to unsuccessful vaccine development, Ngo has rapidly become resistant to almost all classes of antibiotics. Recent data from the Center for Disease Control stated over 500,000 drug resistant Ngo infections occur each year in the United States. Due to this widespread resistance, the current recommended treatment option has become limited to a single drug of last resort: ceftriaxone. However, strains resistant to this drug have now emerged and the disease will likely become untreatable in the near future. This has created a desperate need for the development of novel antibiotics and vaccines in order to fight this disease. Like many bacterial pathogens, Ngo is Gram-negative having both an inner and outer membrane. Outer membrane proteins (OMPs) have been recently identified as a novel class of antimicrobial targets. The biogenesis of these $\beta$-barrel OMPs is mediated by a multi-component protein complex, known as the $\beta$-barrel assembly machinery (BAM) complex. Conserved across all Gram-negative bacteria, BAM is required for viability and represents a powerful potential antibiotic or vaccine target. In E. coli, this $200 \mathrm{kDa}$ complex is comprised of five proteins: BamA, an OMP itself, and four lipoproteins, BamB through E. A clear mechanism for how substrate OMPs are folded and inserted into the membrane by BAM is still poorly understood. My research focuses on understanding the mechanism of BAM from Ngo (NgBAM). Previous studies have indicated that Neisseria do not possess a homolog of BamB in their genome and $\mathrm{NgBAM}$ may function as a four component complex. We are currently working to characterize NgBAM using a combination of cryo-electron microscopy, in vivo studies, and double electron- electron resonance (DEER) spectroscopy. We recently determined the cryo-EM structure of $\mathrm{NgBAM}$ to $6.5 \AA$. While we are working to improve the resolution, secondary structure features were visible and allowed us to build a model of the complex using existing crystal structures of the individual components. Our structural studies have revealed interesting features distinct from E. coli and these observations may point to mechanistic features of the complex unique to Neisseria. In the near future, we will begin to complement this work with in-vivo functional studies and DEER spectroscopy. This work will lay the foundation for characterizing the complex for future vaccine and drug development against gonorrhea. 